\title{
Ist Suchtprävention generell wirtschaftlich?
}

\author{
Is Prevention of Substance Abuse always Efficient?
}

Jürgen Rehm

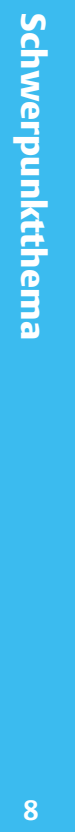

\section{Zusammenfassung}

Prävention wird oft per se als positive gesundheitspolitische Maßnahme im Suchtbereich betrachtet. An verschiedenen Beispielen wird aufgezeigt, dass große präventive Maßnahmen im Suchtbereich oft nicht den gewünschten Erfolg zeigen, in Einzelfällen sogar das Gegenteil dessen bewirken können, was intendiert war. Deshalb wird gefordert, die üblichen Standards der „evidence-based medicine“ auch für Prävention anzuwenden und präventive Aktivitäten im Suchtbereich hinsichtlich Effektivität und Kosteneffektivität zu evaluieren. Gegenargumente zu dieser Forderung werden diskutiert.

\section{Schliisselwörter}

Sucht · Prävention · Effektivität · Kosteneffektivität · Evaluation · evidence-based medicine

\section{Abstract}

Prevention of substance abuse is often seen as a positive health policy per se. Several examples are given, however, where large preventive measures in the field of substance abuse did not show the intended result; some were even associated with the opposite of the intended effects. Thus, the usual standards of evidence-based medicine should also be applied to prevention, and preventive activities in the field of substance abuse should be evaluated for their effectiveness and cost-effectiveness. Counterarguments to this position are discussed.

\section{Key words}

Substance abuse - prevention - effectiveness - cost effectiveness · evaluation $\cdot$ evidence-based medicine

\section{Hintergrund}

Prävention gilt rundum als positiv. Alte Sprichwörter (siehe oben) schwärmen bereits von der Kosteneffektivität proaktiver Prävention im Vergleich zu therapeutischen Verfahren, die WHO empfiehlt Prävention als zugleich effektiv, human und kostengünstig und kein Politiker lässt es sich nehmen, seinen Namen bei passender oder unpassender Gelegenheit mit Präventionskampagnen zu verbinden. Dies gilt nicht zuletzt auch für den Bereich der Substanzmittelabhängigkeit und für Kampagnen wie „Keine Macht den Drogen!“
Dennoch stellt sich die Frage, inwieweit Prävention, so wie sie heute vielfach betrieben wird, etwas nützt und, sofern diese Frage bejaht werden kann, ob der Nutzen in einem sinnvollen Verhältnis zum Aufwand steht. Ehe wir diese Frage zu beantworten suchen, wollen wir kurz auf einige Begrifflichkeiten zum Thema Prävention eingehen.

\section{Die vier Säulen der Pråvention}

Die WHO unterscheidet vier Stufen der Prävention (Tab.1) [1] Wir wollen hier vorwiegend auf die Stufen zwei und drei eingehen: Primär- und Sekundärprävention, wobei der Schwerpunkt auf Stufe zwei gelegt wird. 
Tab. 1 Die vier Stufen der Prävention

\begin{tabular}{lll}
\hline Präventionsstufe & Krankheitsstadium & Zielgruppe \\
\hline Primordialprävention & Ausgangsbedingungen, die zur Kausalität führen & gesamte Bevölkerung und ausgewählte Gruppen \\
\hline Primärprävention & bestimmte Kausalfaktoren & gesamte Bevölkerung, ausgewählte Gruppen und gesunde Personen \\
\hline Sekundärprävention & Frühstadium der Krankheit & Patienten \\
\hline Tertiärprävention & Spätstadium der Krankheit (Behandlung, Rehabilitation) & Patienten \\
\hline
\end{tabular}

Dabei wird kein Überblick über alle präventiven Aktivitäten angestrebt. Vielmehr sollen typische Präventionsaktivitäten wie schulische Prävention oder Medienkampagnen exemplarisch behandelt werden.

\section{D.A.R.E. say no}

D.A.R.E. steht für Drug Abuse Resistance Education (initiiert in den USA in den 80er-Jahren) und bezeichnet wohl das Präventionsprogramm im Drogenbereich, für das weltweit am meisten Geld ausgegeben wird: nach Auskunft der Organisatoren allein 215 Mio. US\$ für die schulische Vermittlung, alles in allem wohl zwischen 1,0 und 1,3 Mrd. US\$ pro Jahr [2]. Nicht weniger als 30000 Polizeibeamte kommen im Rahmen des D.A.R.E.-Programms jedes Jahr in amerikanische Schulen und machen dort strukturierte Prävention. Mit welchem Erfolg? Clayton et al. [3] haben die Effektivität des Programms nach 5 Jahren in einem experimentellen Versuchsplan gemessen und kommen zu dem ernüchternden Schluss, dass sich hinsichtlich des Konsums legaler und illegaler Drogen weder ein Jahr nach Programmende noch über einen 5-Jahres-Zeitraum signifikante Unterschiede zwischen Versuchs- und Kontrollgruppe zeigten. Es ließen sich zwar kurzfristige Wissenseffekte nachweisen, die im Verlauf aber wieder verschwanden. Eine Vielzahl weiterer Evaluationen von D.A.R.E. hat zu ähnlichen Ergebnissen geführt und die Verantwortlichen haben daraufhin 2001 die zugrunde liegende Strategie, das Curriculum sowie die Zielgruppe geändert (von Fünftauf Siebtklässler und von einem auf Wissen basierten Programm hin zu einer Fokussierung auf soziale Normen [4]).

Über die Effektivität des neuen Programms können naturgemäß noch keine längerfristigen Daten vorliegen. Dennoch kann hier bereits jetzt beispielhaft das allgemeine Fazit gezogen werden, dass Prävention keineswegs allgemein wirkt. Und sicherlich will niemand für relativ wirkungslose Prävention mehr als $1 \mathrm{Mrd}$. US\$ jährlich ausgeben; es sei denn, man verfolgt andere politische Ziele als die Reduktion von Substanzmittelgebrauch.

\section{Systematische Evaluation von Präventionsaktivitäten im Bereich Alkohol}

Edwards et al. [5] sowie Babor et al. [6] fassen die entsprechende Literatur zu Schul- und Massenmedienkampagnen im Bereich Alkohol folgendermaßen zusammen:

\section{Schulkampagnen}

„In sum, these well-designed evaluations suggest that even comprehensive school-based prevention programs may not be sufficient to delay the initiation of drinking, or to sustain a small reduction in drinking beyond the operation of the program. Reduced drinking was found when coupled with community interventions, especially those that were successful in reducing alcohol sales and provision of alcohol to youth. More research is needed to confirm the findings of these early studies, which couple school education with family and community interventions, including local policy initiatives [6].“

\section{Massenmedienkampagnen}

„Most evaluations of the impact of mass media campaigns have, instead, typically employed quasi-experimental designs to look for relatively short-term effects of campaigns on the audience. Several reviews were carried out on the evaluations of mass media campaigns conducted in the $1970 \mathrm{~s}$ and $1980 \mathrm{~s}$. They concur in finding that most campaigns showed limited effect on the recipients' beliefs and attitudes, and no impact on self-reported drinking ([7-10]; cited from [5])."

„Previous reviews point to the limited impact on alcohol use and alcohol-related problems from mass-media interventions [6]."

An dieser Stelle darf schon einmal ein Zwischenfazit gezogen werden: Ganz offensichtlich gibt es viele präventive Aktivitäten im Bereich des Suchtmittelgebrauchs, die keinerlei messbare Folgen haben.

\section{Beispiele von Prävention mit messbaren Ergebnissen}

Nach dem oben offerierten Zwischenfazit stellt sich nun die Frage, ob es denn nun Präventionsaktivitäten gibt, die nachweisbar intendierte Ergebnisse zeigen, und zwar möglichst nicht nur auf der Ebene von Konsumrückgang, sondern auf der Ebene substanzbedingter Folgen. In den erwähnten Überblickswerken finden sich für Alkohol durchaus solche Aktivitäten wie z.B. Besteuerung, Verringerung der Dichte von Alkoholverkaufsstellen, zufällige Verkehrskontrollen oder Schulung von Personal in Bars [5, 6]. Allen diesen Maßnahmen ist gemein, dass sie auf die Umgebung des Substanzgebrauchs und nicht direkt auf die beteiligten Personen zielen und zum Teil aus diesem Grund in der Allgemeinbevölkerung nicht unbedingt mit Prävention in Verbindung gebracht werden; mit anderen Worten, sie gelten als nicht unbedingt typisch für Prävention. Und für diese Maßnahmen zeigt sich dann in verschiedenen Beispielen, aber auch in zusammenfassenden Metaanalysen, dass sie im Vergleich zu Be- 
handlungen kosteneffektiv sind, d.h., dass für die gleichen Kosten mehr Nutzen hinsichtlich Krankheitsbelastung (z.B. gemessen in „disability adjusted life years“, DALYs, siehe [11] für eine Übersicht zu substanzgebrauchsbedingter Krankheitsbelastung) erreicht werden kann. Es bleibt dem Leser überlassen, über die Gründe zu spekulieren, warum häufig ineffektive oder nicht evaluierte Präventionsmaßnahmen nachweislich wirksamen Interventionen wie Besteuerung vorgezogen werden.

Bisher war die Rede vorwiegend von Primärprävention. Es gibt selbstverständlich auch empirisch effektive sekundärpräventive Aktivitäten, z.B. so genannte Kurzinterventionen („brief interventions" [6]; siehe auch den Beitrag von Rey-Riek et al. in diesem Schwerpunkt [12]). Mit anderen Worten: Die Schlussfolgerungen hinsichtlich Primärprävention gelten auch hinsichtlich Sekundärprävention im Bereich Alkohol: Bestimmte Programme erzielen messbar positive Ergebnisse, andere sind nicht evaluiert und wieder andere erzielen keine Ergebnisse. Selbstverständlich sind auch Fälle denkbar, wo Prävention negative Ergebnisse verursacht, d.h. Ergebnisse, die in entgegengesetzte Richtung wie intendiert gehen.

\section{Diskussion}

Da die Ergebnisse von herkömmlicher Prävention im Suchtbereich also nachweislich in verschiedene Richtungen gehen (intendierte, keine und nicht intendierte Wirkungen), gibt es keinen Grund, warum sie nicht wie andere Maßnahmen im Gesundheitswesen auch den üblichen Prinzipien von „evidence-based medicine" unterworfen werden sollten. Mit anderen Worten: Präventive Aktivitäten sollten ebenso evaluiert werden wie Behandlung. Genauso sollten hinsichtlich der Mittelvergabe Prinzipien der Kosteneffektivität von Maßnahmen in das Gesamtkalkül mit einbezogen werden. Eine Mittelvergabe, die nur auf Kosteneffektivität basiert, kann es dabei nicht geben, denn man muss ethisch immer das Gesamtsystem im Auge behalten. Selbst wenn eine bestimmte Kombination präventiver Aktivitäten also effizienter suchtmittelbedingte Krankheitsbelastung reduziert als Behandlung, ist es aus ethischen Gründen fragwürdig, alle Mittel in diese Aktivitäten fließen zu lassen, falls es in der jeweiligen Gesellschaft schon Personen gibt, die erkrankt sind, d.h., denen durch präventive Aktivitäten nicht mehr geholfen werden kann. Kosteneffizienz kann also nur ein, wenn auch wichtiger Gesichtspunkt bei der Auswahl gesundheitspolitischer Maßnahmen sein.

Zum Schluss soll auch noch ein häufiges Gegenargument zur Evaluation von präventiven Tätigkeiten diskutiert werden. Dieses Argument tritt in verschiedenen Formen auf, aber im Kern besagt es, dass es Ziel verschiedener präventiver Maßnahmen wie Schulerziehung oder massenmedialer Kampagnen sein muss, ein kulturelles Klima zu schaffen, in dem dann andere, effektivere Maßnahmen erst durchgeführt werden können. Nach diesem Argument sind Evaluationen von entsprechenden präventiven Maßnahmen, die Erfolge im Verhalten suchen, fehlgeleitet.

Dazu zwei Punkte, wieder exemplifiziert am Beispiel von Alkoholmaßnahmen: Das Argument beruht auf einem angenommenen Wirkmechanismus, der zweistufig ist. Erstens wird ein kulturelles Klima geschaffen, in dem zweitens dann bestimmte andere Mechanismen effektiver sind. Nichts steht einer empirischen Evaluation dieser Wirkmechanismen entgegen: Es müsste zunächst nachgewiesen werden, dass die Kampagne entsprechende Meinungen, Überzeugungen und Attitüden in der Allgemeinbevölkerung nachhaltig verändert. Dann sollte nachgewiesen werden, dass die Effektivität von empirisch erfolgreichen Maßnahmen mit den entsprechenden Meinungen, Überzeugungen oder Attitüden kovariiert. Allerdings ist klar, dass beide Stufen erfolgreich sein müssen, um den Gesamterfolg zu belegen.

Zum Zweiten bleibt selbst bei Nachweis eines Wirkungseintritts beider Faktoren immer noch die Frage nach deren Kosteneffektivität. Wenn andere präventive Maßnahmen wie Steuererhöhung nachweislich effektiv in der Reduktion von alkoholbedingten Schäden sind, müssten die alternativen Maßnahmen weniger kosten und gleich wirksam sein oder gleich kosten und insgesamt wirksamer sein. Hier scheinen zumindest Zweifel angebracht, die allerdings erst durch konkrete empirische Daten fundiert bzw. ausgeräumt werden können.

Schließlich bleibt noch das Argument, dass nachweislich wirksame Maßnahmen wie Besteuerung nur dann durchgesetzt werden können, wenn vorher der Boden durch andere, nicht nachweislich wirksame Maßnahmen wie massenmediale Kampagnen bereitet wurde. Dieses Argument wurde unseres Wissens nach bislang noch nie empirisch belegt. Im Gegenteil: Im Tabakbereich wurden in vielen Ländern Gesetze beschlossen, wie z.B. Verbote des Rauchens an öffentlichen Plätzen, die durchaus wirksam waren und erst später die Zustimmung einer Mehrheit der Bevölkerung erfuhren.

Insgesamt bleibt also festzuhalten, dass präventive Maßnahmen in jedem Fall evaluiert werden sollten, sei es hinsichtlich der intendierten Ergebnisse oder hinsichtlich eines Wirkmechanismus, der irgendwann später nachweislich zu Ergebnissen führen sollte. Ein Verweis auf angebliche Nichtmessbarkeit oder gar prinzipiell nicht messbare Konzepte ist nicht ausreichend oder vertretbar - speziell in einer Zeit, in der eine allgemeine Knappheit von Ressourcen herrscht und der Nachweis von Effektivität oder sogar Kosteneffektivität zu einem allgemein akzeptierten Standard geworden ist.

\section{Literatulr}

${ }^{1}$ Beaglehole R, Bonita R, Kjellström T. Einführung in die Epidemiologie. Bern: Verlag Hans Huber 1997

2 Shepard EM. The Economic Costs of D.A.R.E. Syracuse, NY: Institute of Industrial Relations 2001

${ }^{3}$ Clayton RR, Cattarello AM, Johnstone BM. The effectiveness of Drug Abuse Resistance Education (project DARE): 5-year follow-up results. Prev Med 1996; 25 (3): 307-318

${ }^{4}$ www.drugpolicy.org/news/02_20_01dare2.cfm. (29.1.2003)

${ }^{5}$ Edwards G, Anderson P, Babor TF et al. Alcohol Policy and the Public Good. Oxford: Oxford University Press 1994

${ }^{6}$ Babor T, Caetano R, Casswell S et al. No Ordinary Commodity: Alcohol and Public Policy. Oxford: Oxford University Press (in press)

${ }^{7}$ Blane HAT, Hewitt LE. Alcohol, public education and mass media: an overview. Alcohol, Health and Research World 1980; 5: 2-16

${ }^{8}$ Wallack LM. Assessing effects of mass media campaigns: an alternative perspective. Alcohol, Health and Research World 1980; 5: 17-29 
${ }^{9}$ Dorn N, South N. Message in a Bottle: Theoretical Overview and Annotated Bibliography on the Mass Media and Alcohol. Aldershot: Gower 1983

${ }^{10}$ Moskowitz JM. The primary prevention of alcohol problems: a critical review of the research literature. Journal of Studies on Alcohol 1989; 50: $54-88$
${ }^{11}$ World Health Organization. World Health Report 2002: reducing risks, promoting healthy life. Geneva: WHO 2002

12 Rey-Riek S, Güttinger F, Rehm J. Lohnt sich betriebliche Suchtprävention? Zu Effektivität und Effizenz betrieblicher Alkoholprävention. Suchttherapie 2003; 4: 19-24 